\title{
Corrosion cast study of the canine hepatic veins
}

\author{
M. Uršič, M. Vrecl, G. Fazarinc \\ Institute for Anatomy, Histology and Embryology, Veterinary Faculty, Ljubljana, Slovenia \\ [Received 14 March 2014; Accepted 26 April 2014]
}

\begin{abstract}
This study presents a detailed description of the distribution, diameters and drainage patterns of hepatic veins on the basis of the corrosion cast analysis in 18 dogs. We classified the hepatic veins in three main groups: the right hepatic veins of the caudate process and right lateral liver lobe, the middle hepatic veins of the right medial and quadrate lobes and the left hepatic veins of both left liver lobes and the papillary process.

The corrosion cast study showed that the number of the veins in the Nomina Anatomica Veterinaria and most anatomical textbooks is underestimated. The number of various-sized hepatic veins of the right liver division ranged from 3 to 5 and included 1 to 4 veins from the caudate process and 2 to 4 veins from the right lateral liver lobe. Generally, in all corrosion casts, one middle-sized vein from the right part of the right medial lobe, which emptied separately in the caudal vena cava, was established. The other vein was a large-sized vein from the remainder of the central division, which frequently joined the common left hepatic vein from the left liver lobes. The common left hepatic vein was the largest of all the aforementioned hepatic veins. (Folia Morphol 2014; 73, 4: 475-481)
\end{abstract}

Key words: liver, veins, branching pattern, dog

\section{INTRODUCTION}

The development of liver surgery is based on improved knowledge of the internal liver structures, above all the organisation, courses and ramifications of the liver vessels [1]. Such approaches can provide surgeons with useful information and knowledge for the safe removal of affected liver parts. Detailed knowledge of the liver vessel anatomy is also crucial to understanding the spatial relationships among liver structures that enable correct interpretation/recognition of (i) radiographic and computed tomography scans, (ii) ultrasound images and (iii) intrahepatic vascular anomalies [9], including extra- or intrahepatic left- and right-sided vessel shunts [4, 11, 12] and patent venous ducts [17]. Research into hollow liver structure arrangement has been based on the studies of formalin-fixed specimens, angiograms and corrosion casts. The latter has enabled the determination of the 3-dimensional vessel arrangement, which is of primary importance for analysing the anatomical variations in vascular anatomy within the organs $[1,4]$.

Deep fissures divide the canine liver into 6 distinct lobes (left lateral, left medial, quadrate, right medial, right lateral and caudate); according to Sleight and Thomford [16], these lobes are arranged into 3 major divisions (Fig. 1). The caudate process and right lateral lobe, which lie completely to the right side, form the right liver division; the right medial lobe and quadrate lobe, which are located mostly on the median plane, constitute the central liver division; and the papillary process and both left hepatic lobes on the left form the left liver division.

The ramifications of the portal veins and hepatic arteries in dogs have been described in detail in some previous studies $[8,15,18]$. However, the intrahepatic courses and ramifications of the hepatic veins have

Address for correspondence: Dr M. Uršič, Institute for Anatomy, Histology and Embryology, Veterinary Faculty, Gerbičeva 60, 1000 Ljubljana, Slovenia, tel: +38614779100, fax: +38612832243, e-mail: matjaz.ursic@vf.uni-lj.si 


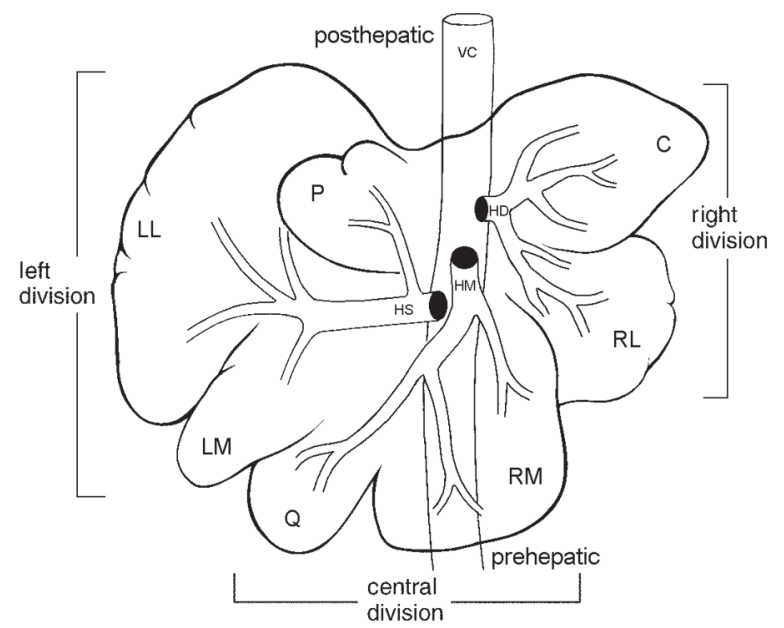

Figure 1. Schematic representation of the three major divisions, adapted from Sleight and Thomford (1970), and the individual lobes of the canine liver. The variations of the hepatic veins and tributaries, adapted from the NAV (2005), are depicted; C - caudate process, $\mathrm{LL}$ — left lateral, $\mathrm{LM}$ — left medial, $\mathrm{RL}$ — right lateral, $\mathrm{RM}$ — right medial, $\mathrm{O}$ - quadrate, $\mathrm{P}$ - papillary process, $\mathrm{VC}-\mathrm{v}$. cava caudalis, HD - v. hepatica dextra, HM - v. hepatica media, HS v. hepatica sinistra; black ellipses indicate hepatic vein openings.

not been sufficiently analysed. For this reason, the objective of this study was to provide descriptions of the anatomy of the hepatic veins in dogs. Anatomical details regarding the localisations, numbers and diameters of the hepatic veins could be used as possible aids in both the interpretation of canine liver images and surgical management.

\section{MATERIALS AND METHODS}

This study was conducted on 18 corrosion casts obtained from conditioned dogs of both sexes and various breeds. The dogs were legally euthanised in accordance with the Slovenian Animal Protection Act (Official Gazette of the Republic of Slovenia, No. 43/2007), which is in harmony with the Council Directive 86/609/EEC, for reasons unrelated to our study. The Veterinary Administration of the Republic of Slovenia approved our gross anatomy laboratory for anatomical preparation (registration number SI-B-07-14-05). All the investigated livers were undamaged and macroscopically healthy. The livers were removed as units from the cadavers' abdominal cavities with the retrohepatic segment of the caudal vena cava ( $v$. cava caudalis), which lies in a groove between the caudate lobe and the right lateral lobe. The caudal vena cava was ligated cranial to the hepatocaval confluences and was then transected caudal to the liver caudate process (processus caudatus). The methods for cre-

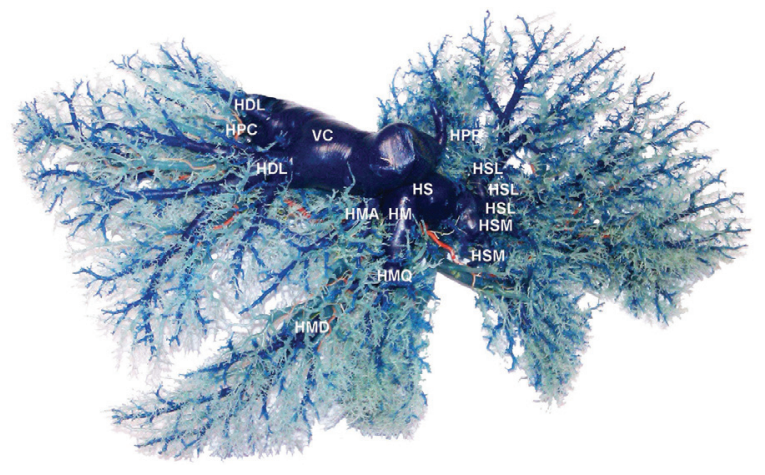

Figure 2. The diaphragmatic side of the liver corrosion cast (dark blue - hepatic vein system; light blue — portal vein system; red — hepatic artery system); VC — v. cava caudalis; HPC — vv. hepaticae processus caudati; $\mathrm{HDL}-v v$. hepaticae dextrae laterales; HMA — v. hepatica media accesoria; HM — v. hepatica media; $\mathrm{HMD}$ - v. hepatica media; HMO — v. hepatica media lobi quadrati; HS — v. hepatica sinistra; HSM — vv. hepaticae sinistrae mediales; $\mathrm{HSL}-v v$. hepaticae sinistrae laterales; HPP $-v$. hepatica processus papillaris.

ating corrosion casts of the liver vessels (as shown in Fig. 2) have been previously reported in detail $[6,7]$. Plastic cannulas were inserted into the bile duct, hepatic artery, portal vein and caudal vena cava. Structures requiring a small volume of mass (i.e., bile ducts and the hepatic artery) were injected first, followed by the portal vein, and the hepatic veins were finally injected through the caudal vena cava. Acrylate monomers, in both powder and solvent forms (Polirepar $\mathrm{S}^{\odot}$; Polident, Dental Products Industry, Slovenia), were mixed in a 2:1 proportion and were injected into the luminal liver structures. Before injection, a polyurethane pigment was added to the compound. To prevent effects on the vessels' diameters, the injection was performed manually, until there was a noticeable increase in the pressure of the injected mass. In 20-30 min, the cast material completely polymerised. These preparations were subsequently placed into $30 \%$ hydrochloric acid solution. A few days later, the specimens were rinsed with water jets, and the disintegrated liver tissue was carefully removed.

The hepatic vein calibres were measured with a slide gauge at the point of confluence. The retrohepatic segment represented the length of the caudal vena cava between the hepatocaval confluences of the most caudal and most cranial hepatic veins. The diameter of the retrohepatic segment was measured above the right lateral lobe.

In our study, we followed the segmentation of the canine liver to describe properly the tributary regions of the hepatic veins. 
Table 1. The numbers and lobar distributions of the hepatic veins and the numbers of the hepatocaval confluences in canine liver casts

\begin{tabular}{|c|c|c|c|c|c|c|c|c|c|c|c|c|}
\hline \multirow[t]{3}{*}{ Liver } & \multicolumn{3}{|c|}{ Right division } & \multicolumn{4}{|c|}{ Central division } & \multicolumn{4}{|c|}{ Left division } & \multirow[t]{3}{*}{ HCC } \\
\hline & \multicolumn{2}{|c|}{ Caudate } & \multirow{2}{*}{$\begin{array}{c}\begin{array}{c}\text { Right } \\
\text { lateral }\end{array} \\
\text { HDL }\end{array}$} & \multicolumn{3}{|c|}{ Right medial } & \multirow{2}{*}{$\begin{array}{l}\text { Quadrate } \\
\text { HM(HS)O }\end{array}$} & \multirow{2}{*}{$\begin{array}{c}\begin{array}{c}\text { Left } \\
\text { medial }\end{array} \\
\text { HSM }\end{array}$} & \multirow{2}{*}{$\begin{array}{c}\text { Left } \\
\text { HS }\end{array}$} & \multirow{2}{*}{$\begin{array}{c}\begin{array}{c}\text { Left } \\
\text { lateral }\end{array} \\
\text { HSL }\end{array}$} & \multirow{2}{*}{$\begin{array}{c}\text { Papillary } \\
\text { HPP }\end{array}$} & \\
\hline & HPC & HDC & & HMA & HMD & HM & & & & & & \\
\hline Var. 1 & \multicolumn{2}{|c|}{1} & 4 & 1 & 1 & & (1) & 2 & 1 & 2 & 1 & 8 \\
\hline Var. 2 & \multicolumn{2}{|l|}{4} & 2 & 1 & 1 & 1 & 1 & 1 & 1 & 2 & 2 & 8 \\
\hline Var. 3 & \multicolumn{2}{|l|}{2} & 4 & 1 & 1 & 1 & 1 & 3 & 1 & 3 & 1 & 8 \\
\hline Var. 4 & \multicolumn{2}{|l|}{2} & 2 & 1 & 1 & 1 & 1 & 3 & 1 & 3 & 1 & 6 \\
\hline Var. 5 & 1 & 1 & 3 & 1 & 1 & $1^{*}$ & 1 & 1 & $1 *$ & 2 & $1 *$ & 7 \\
\hline Var. 6 & \multicolumn{2}{|r|}{1} & 2 & 1 & 1 & 1 & 1 & 2 & 1 & 3 & 1 & 6 \\
\hline Var. 7 & 1 & 1 & $21 *$ & $1 *$ & 1 & $1 * *$ & 1 & 1 & 1 & 2 & $1 * *$ & 7 \\
\hline Var. 8 & 1 & 1 & 2 & 1 & 1 & 1 & 1 & 1 & 1 & 2 & 1 & 6 \\
\hline Var. 9 & 1 & & 3 & 1 & 1 & 1 & 1 & 1 & 1 & 2 & 1 & 6 \\
\hline Var. 10 & & 1 & 2 & 1 & 1 & $1^{*}$ & $1(1)$ & 1 & $1 *$ & 2 & 1 & 5 \\
\hline Var. 11 & 2 & 1 & 3 & 1 & 1 & 1 & 1 & 1 & $1 *$ & 2 & $1^{*}$ & 8 \\
\hline Var. 12 & 1 & & 4 & 1 & 1 & 1 & 1 & 3 & 1 & 3 & 2 & 7 \\
\hline Var. 13 & 1 & & 2 & 1 & 1 & 1 & 2 & 1 & 1 & 1 & 2 & 5 \\
\hline Var. 14 & 1 & & 2 & 2 & 1 & 1 & 1 & 2 & 1 & 3 & 2 & 6 \\
\hline Var. 15 & 2 & & 3 & 1 & 1 & 1 & 1 & 2 & 1 & 3 & 1 & 7 \\
\hline Var. 16 & 1 & & 2 & 1 & 1 & 1 & 1 & 1 & 1 & 3 & 1 & 5 \\
\hline Var. 17 & 2 & & 2 & 2 & 1 & 1 & 1 & 1 & 1 & 3 & 1 & 7 \\
\hline Var. 18 & 2 & 1 & 2 & 1 & 1 & 1 & 1 & 2 & 1 & 3 & 1 & 7 \\
\hline
\end{tabular}

*Indicates veins with the same ostium; bold indicates hepatocaval confluences; HDC — v. hepatica dextra communis; HSQ — v. hepatica sinistra lobi quadrat; HCC — the number of hepatocaval confluences; rest abbreviations as in Figs. 1, 2

\section{RESULTS}

Data on the numbers and lobar distributions of the hepatic veins and numbers of the hepatocaval confluences in 18 canine liver casts are summarised in Table 1, while the characteristic branching patterns are schematically shown in Figure 3. The abdominal segment of the caudal vena cava gradually inclined ventrally before it reached the liver. In all the corrosion casts, the retrohepatic segment was closely related to the liver caudate process and was deeply grooved in the liver surface. The collecting hepatic veins, after uniting with many others, formed the larger hepatic veins. Finally, all of the hepatic veins drained into the retrohepatic segment of the caudal vena cava. In the revised liver corrosive casts, the total number of hepatic veins that drained into the caudal vena cava varied from 5 to 8 (Table 1). The minimum and maximum diameters of the retrohepatic segment of the caudal vena cava and hepatic veins at the point of their outflow are shown in Table 2 . The length of the retrohepatic segment of the caudal vena cava varied from a minimum of 35 to a maximum of $75 \mathrm{~mm}$ (58.1 $\pm 11.3 \mathrm{~mm})$.

\section{Right liver division}

The right hepatic veins ( $v v$. hepaticae dextrae) are the veins that run from the right liver division; the number of veins varied from 3 to 6 (Table 1). These veins originated from the caudate process and the right lateral lobe. The collecting veins ran through the liver lobes in the craniodorsal and left direction and tended to join together and finally drain at different sites of the caudal portion of the retrohepatic segment of the caudal vena cava. Their diameters near their openings ranged from 2 to $14 \mathrm{~mm}(6.7 \pm 3.4 \mathrm{~mm})$ (Table 2, HD).

The openings of the caudate process veins ( $v v$. hepaticae processus caudati) were in the right ventral wall of caudal vena cava and were the most caudal of the hepatic veins' ostia. These veins were located cranially and dorsally to the right portal vein branch and were intertwined with the branches of the portal system. In 1 case, the 2 most caudal veins ( 3 and $5 \mathrm{~mm}$ in diameter) originated from the right lateral lobe and not from the caudate process.

The caudate process veins, which drained the blood only from the caudate process, were present 

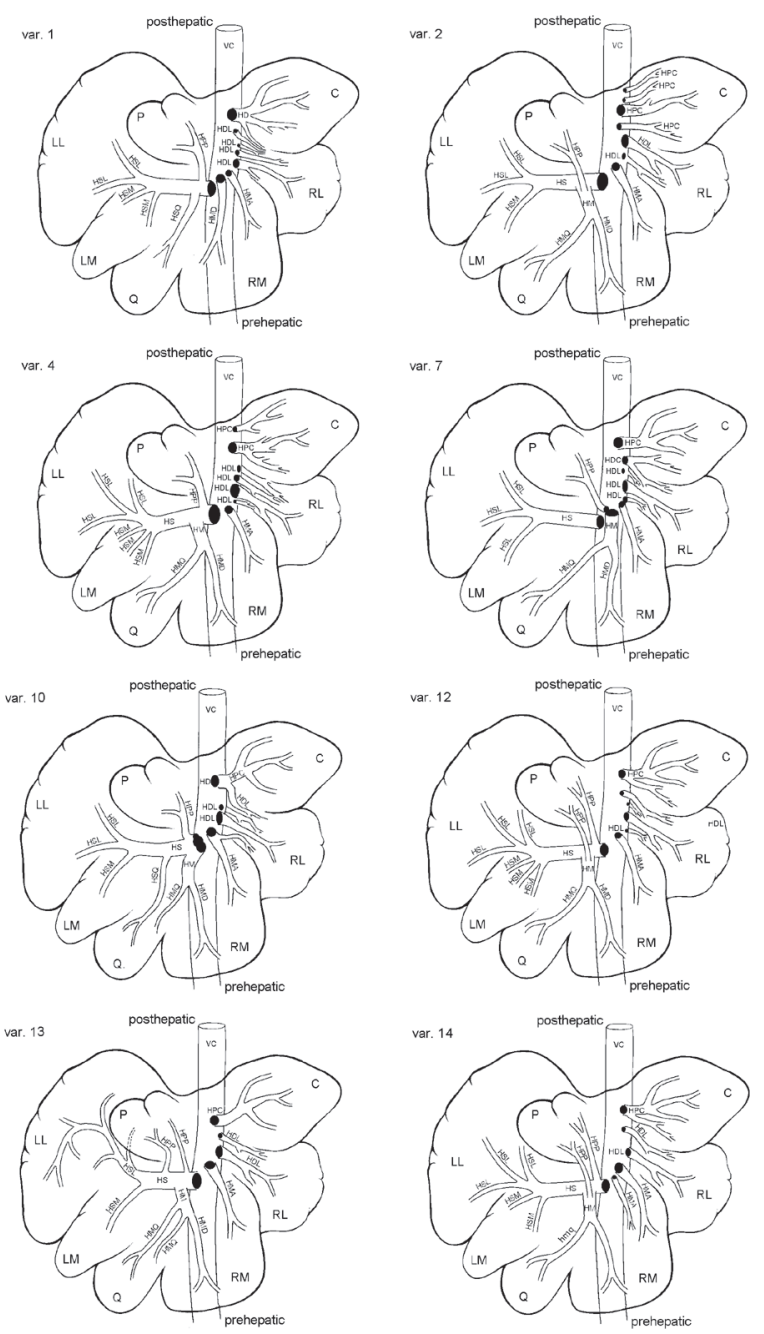

Figure 3. Branching patterns of the canine hepatic vein system. Note the variations in configuration and position relative to the VC. Each drawing represents 1 case; HDC - v. hepatica dextra communis; HSO - v. hepatica sinistra lobi quadrati; black ellipses indicate hepatic vein openings; rest abbreviations as in Figs. 1, 2

in 16 corrosion casts. Only 1 caudate process vein was present in 9 cases (Fig. 3; var. 13). In 4 cases, the caudate process was drained by the caudate process vein and another vein, which fused with the veins from the adjacent part of the right hepatic lobe (Fig. 3 ; var. 7) to form a common right hepatic vein ( $v$. hepatica dextra communis). This vein was visible in the cleft between the caudate process and the right lateral lobe and entered the caudal vena cava directly. In 6 cases, there were 2 caudate process veins (Fig. 3; var. 4). One vein was dominant and wider, with a diameter range of 7 to $14 \mathrm{~mm}$, and the other was evidently smaller (diameter range of 2-4 mm). In 2 cases of double caudate process veins, a common right hepatic vein was also present.
Table 2. The minimum and maximum diameters $[\mathrm{mm}]$ of the retrohepatic segments of the caudal vena cava and the diameters $[\mathrm{mm}]$ of the hepatic veins at the point of their outflow

\begin{tabular}{lccc}
\hline Vein & \multicolumn{3}{c}{ Diameter [mm] } \\
\cline { 2 - 4 } & Min & Max & Mean \pm SD \\
\hline VC & 13 & 26 & $20.9 \pm 3.6$ \\
HPC & 2 & 14 & $6.9 \pm 3.4$ \\
HD & 2 & 14 & $6.7 \pm 3.4$ \\
HDL & 2 & 13 & $6.4 \pm 3.4$ \\
HDC & 4 & 9 & $5.2 \pm 1.9$ \\
HMA & 3 & 7 & $5.0 \pm 1.2$ \\
HM & 7 & 15 & $11.1 \pm 2.0$ \\
HMD & 4 & 12 & $6.8 \pm 2.0$ \\
HO & 3 & 10 & $6.0 \pm 1.7$ \\
HS & 11 & 21 & $14.9 \pm 2.6$ \\
HPP & 3 & 8 & $5.4 \pm 1.6$ \\
HSM & 3 & 9 & $5.6 \pm 1.9$ \\
HSL & 3 & 12 & $7.0 \pm 2.1$ \\
\hline
\end{tabular}

$\mathrm{HDC}-v$. hepatica dextra communis; $\mathrm{HO}-v$. hepatica lobi quadrati; rest abbreviations as in Figs. 1, 2

Four caudate process veins were found in 1 corrosion cast (Fig. 3; var. 2): 1 wider (6 $\mathrm{mm}$ in diameter) and 3 smaller, with mean diameter of $3.3 \pm 1.2 \mathrm{~mm}$.

In 2 corrosion casts, blood from the caudate process was drained by the vein, which also drained a part of the right lateral lobe (Fig. 3; var. 10). Their diameters were $11 \mathrm{~mm}$ and $12 \mathrm{~mm}$, respectively. An additional 2 or 4 right lateral hepatic lobe veins were also present.

Altogether, in 6 of all the examined cases, a single short common right hepatic vein with mean diameter $5.2 \pm 1.9 \mathrm{~mm}$ (Table 2, HDC) was located in the cleft between the caudate process and the right lateral lobe (Fig. 3; var. 7).

The lateral right liver lobe veins ( $v v$. hepaticae dextrae laterales) were located in the right side of the caudal vena cava and were present in all the corrosion casts. They emptied into the caudal vena cava, near and slightly cranial to the caudate process veins. Two veins from the lateral right liver lobe were present in 10 corrosion casts (Fig. 3; var. 13). In 5 corrosion casts, 3 lateral right hepatic veins were observed (Fig. 3; var. 7). Four lateral right hepatic veins were present in 3 of the examined corrosion casts (Fig. 3; var. 1 and var. 12). In 16 cases, 1 lateral right hepatic vein was thicker (diameter of 8 to $13 \mathrm{~mm}$ ), while the other veins were thinner and drained smaller peripheral parts of the lateral right liver lobe. $\ln 2$ cor- 
rosion casts, 2 similarly thick lateral right hepatic veins were noted (diameters of 10 and $8 \mathrm{~mm}$ and 6 and $5 \mathrm{~mm}$, respectively). Additionally, in 2 cases, 1 of the lateral right hepatic veins of the medial part of the right lateral lobe (diameter of $4 \mathrm{~mm}$ ) joined a middle hepatic accessory vein ( $v$. hepatica media accessoria) (Fig. 3; var. 10).

\section{Central liver division}

The middle hepatic accessory vein ( $v$. hepatica media accessoria) was present in all the casts and measured 3 to $7 \mathrm{~mm}$ in diameter $(5.0 \pm 1.2 \mathrm{~mm})$, as shown in Table 2 (HMA). In 14 cases, the middle hepatic accessory vein diameter was equal to or greater than $5 \mathrm{~mm}$. The vein ran in the dorsocranial direction and slightly to the left and was located near the cranial surface of the right medial lobe. In each corrosion cast, the vein originated from the right part of the right medial lobe and was located ventral to the retrohepatic segment of the caudal vena cava. The vein drained into the cranial part of the retrohepatic segment from the ventral aspect near the hepatocaval confluence of the left and middle hepatic veins. The middle hepatic accessory vein was single in 16 corrosion casts (Fig. 3, except var. 14). In 2 casts, 2 small-sized middle hepatic accessory veins (3 $\mathrm{mm}$ in diameter) were noted (Fig. 3; var. 14). In 2 cases, the middle hepatic accessory vein also received 1 vein from the medial part of the right lateral lobe (Fig. 3; var. 10).

The middle hepatic vein ( $v$. hepatica media) was the main vein from the central liver division, and it passed through the base of the quadrate lobe. In all the cases, this vein consisted of a single venous trunk formed by fusion of the collecting veins from the left part of right medial and whole quadrate lobes. Its diameter ranged from 7 to $15 \mathrm{~mm}(11.1 \pm 2.0 \mathrm{~mm}$; Table 2, HM). In 15 cases, the middle hepatic vein joined the left hepatic vein from the ventrocaudal direction. In 2 corrosion casts, both of the main veins from the central and left divisions had a common hepatocaval confluence in the left ventral surface of the retrohepatic segment (Fig. 3; var. 10).

Usually, the 2 main collecting veins of the liver central division formed the middle hepatic vein. One vein was the middle right hepatic vein ( $v$. hepatica media dextra) on the right side, which originated from the left part of the right medial lobe and had a diameter of 4-12 mm (6.8 $\pm 2.0 \mathrm{~mm}$; Table 2, HMD). This vein ran to the left and joined the collecting vein from the quadrate lobe. The other quadrate lobe vein had a diameter of 3-10 mm (6.0 $\pm 1.7 \mathrm{~mm}$; Table 2, HQ) and ran in the craniodorsal direction. In 1 corrosion cast, the middle right hepatic vein was a thick single vein (12 $\mathrm{mm}$ in diameter) that opened directly into the cranial part of the retrohepatic segment of the caudal vena cava (Fig. 3; var. 1). In this case, the vein from the quadrate lobe ( $v$. hepatica sinistra lobi quadrati) emptied separately into the left hepatic vein (Fig. 3; var. 1). In 2 corrosion casts, 2 distinctive collecting hepatic veins from the quadrate lobe were present. In 1 case, 1 vein ( $v$. hepatica media lobi quadrati) drained into the middle hepatic vein, and the other, like the $v$. hepatica sinistra lobi quadrati, drained into the left hepatic vein (Fig. 3; var. 10). Alternatively, both veins from the quadrate lobe joined the middle right hepatic vein (Fig. 3; var. 13).

\section{Left liver division}

Blood from the left lobes was collected via the left hepatic vein ( $v$. hepatica sinistra), which was the thickest venous trunk, with a diameter ranging from 11-21 mm (14.9 $\pm 2.6 \mathrm{~mm}$ ) (Table 2, HS). The left hepatic vein was created by fusion of the collecting veins from both of the left lobes. The collecting veins converged inside each of the left lobes and joined to form the common large venous trunk, which was visible within the cleft between the left lateral and medial lobes. The left hepatic vein ran obliquely to the right side toward the caudal vena cava. Close to the hepatocaval confluence, the vein usually merged with the middle hepatic vein and entered the retrohepatic segment from the left aspect. This hepatocaval confluence was the most cranial and was situated near the diaphragm (Fig. 3).

In 9 corrosive casts, 3 main lateral left hepatic veins (Fig. 3; var. 4, 12 and 14) were located in the left lateral lobe, and in 8 casts, 2 veins converged (Fig. 3; var. 1, 2, 7 and 10). In 1 corrosive cast, 1 large lateral left hepatic vein, with a diameter of $11 \mathrm{~mm}$, arched at the central position of the left lateral lobe and continued directly to the left hepatic vein (Fig. 3; var. 13).

In all the casts, the medial left hepatic veins ran parallel to the left medial lobe portal vein branches. The number of medial left hepatic veins varied from 1 to 3. Ten cases had only 1 (Fig. 3; var. 2, 7, 10 and 13), 5 cases had 2 (Fig. 3; var. 1 and 14) and 3 cases had 3 left medial hepatic veins (Fig. 3; var. 4 and 12).

Papillary process veins ( $v v$. hepaticae processus papillaris) were situated cranial to the papillary process 
branches of the portal vein. In 14 corrosion casts, the papillary process vein was a single vein, which usually drained into the left hepatic vein on its visceral aspect (10 cases, Fig. 3; var. 1 and 4), or it rarely emptied into the middle hepatic vein (1 case, Fig. 3; var. 2). In 2 corrosion casts, the middle, left and papillary process veins merged together into a short trunk, which entered into the caudal vena cava (Fig. 3; var. 10). In 1 cast, the papillary process vein drained directly into the left surface of the caudal vena cava (Fig. 3; var. 7). In 4 casts, there were 2 papillary process veins, which drained separately into the middle or left hepatic veins (Fig. 3; var. 12, 14 and 13).

\section{DISCUSSION}

Detailed knowledge of the hepatic vascular anatomy is essential for the interpretation of radiological and ultrasound images and computed tomography scans and for advances in liver surgery. However, the anatomy of the major hepatic veins in dogs has been less extensively studied, and to our knowledge, no actual systematic studies of the hepatic veins' branching patterns have been performed. Using the corrosion cast method, which provides a good 3-dimensional view of the hollow liver structures and their spatial correlations, we aimed to provide a detailed description of the anatomic variations of the hepatic veins and to increase the comprehension of the canine hepatic vascular anatomy.

In the Nomina Anatomica Veterinaria (NAV) [14] and in some anatomical textbooks [4, 5, 10, 13], only 3 hepatic veins (Fig. 1), which drain different liver divisions, are mentioned ( $v$. hepatica dextra, media and sinistra). Wittleben [19] and Carlisle and Heath [2] presented more precise descriptions of the canine hepatic veins. Wittleben [19] had already described 4 hepatic veins, which enter the caudal vena cava separately, and designated them with different terms: $v$. hepatica dextra accessoria of the caudate process, $v$. hepatica dextra of the right lateral lobe, $v$. hepatica media of the right medial and quadrate lobes and large $v$. hepatica sinistra, which drain venous blood from both left lobes and the papillary process. Carlisle and Heath [2] reported patterns of venous branching to each lobe of the liver with little variation between individuals. All the liver lobes have definite veins so that the left lateral and left medial, quadrate, right medial and right lateral, caudate and papillary veins can be distinguished; however, the pattern of their merging before they enter the caudal vena cava was not described. In our study, according to the NAV [14], each of the veins formed by the fusion of collecting veins was designated as the hepatic vein (right, middle and left). We also used some additional anatomical terms for the liver's outgoing system of hepatic veins, which were not described in the NAV [14] or in anatomical textbooks $[4,5,10,13]$.

In comparison to the portal vein system, the position of the collecting hepatic veins was on the diaphragmatic side, while the portal vein system branches were usually located toward the visceral side of the liver. The smaller branches of both venous systems crossed each other and were intertwined.

The caudate process veins and right lateral veins entered the caudal vena cava as the first veins from the caudal direction. In a majority of cases, the veins from the caudate process and the right lateral lobe drained into the caudal vena cava with 2 or more veins. The diameters of these veins were large in scale, and the minor veins usually drained more marginal lobar parts. It can be concluded that the numbers, positions and diameters of the veins from the right liver division are less consistent. Carlisle and Heath [2] and Covey et al. [3] reported that the right lateral lobar hepatic veins drained directly via 1 to 3 veins and that the caudate process had 1 or 2 hepatic veins that drained directly into the caudal vena cava. Sleight and Thomford [16] reported that several tiny hepatic veins entered the caudal vena cava, where its wall was contiguous with the hepatic parenchyma. Our observations were largely in accordance with the abovementioned reports. However, we observed a greater total number of small- or medium-sized veins ( 3 to $6 \mathrm{~mm}$ ), which emptied into the caudal vena cava. This finding contradicts the NAV [14], which stated that the veins from the right liver division joined to form the right hepatic vein (Fig. 1). The small-sized veins are of little anatomical importance, but they could be sources of troublesome bleeding during surgeries due to their variability in location. During surgery, such as partial liver resections, these veins must be found and isolated.

The hepatic veins lead separately from smaller parts of 2 adjacent lobes. The latter are often small veins, formed by the merger of collecting veins from both the caudate process and the right lateral lobe, which can be partly fused in dogs $[5,13]$.

The number and arrangement of the hepatic veins, which drain the central division, were more consistent, with only small variations between the 
observed corrosion casts. Collecting veins from the lateral part of the right medial lobe were oriented dorsally and usually merged into the middle accessory hepatic vein. In comparison to the large divisional veins, the accessory hepatic vein in our cases represented a medium-sized vein. The accessory hepatic vein (occasionally 2 ) was present in all the cases as a separate vein and was found on the visceral surface of the right medial lobe. As reported by Covey et al. [3], the accessory hepatic vein might also be visible as an indentation of the caudal vena cava on the diaphragmatic surface. This vein has not been mentioned in either anatomy textbooks $[4,5,10,13]$ or the NAV [14]. Thus, it could be inaccurately interpreted that blood from the aforementioned liver part enters the caudal vena cava via the middle hepatic vein. The greater left part of the central division is drained mostly by the medium-sized right medial and quadrate lobe veins, which form a $V$ shape in the bed of the gallbladder [2]. These veins usually join into a short and large-sized middle hepatic vein. The middle hepatic vein mainly joins the left hepatic vein, while according to the NAV [14]; the middle hepatic vein empties directly into the caudal vena cava (Fig. 1).

The left liver division was drained by a common large venous trunk. Several medium-sized or larger collecting veins ran obliquely from the left liver lobes and united with each other to form the large-sized left hepatic vein, whose course was straight and was directed toward the left aspect of the caudal vena cava. The papillary process vein ( 1 or rarely 2 ) entered the caudal vena cava via the left or middle hepatic vein and almost never entered separately.

\section{CONCLUSIONS}

In summary, we identified several significant hepatic veins, which entered the caudal vena cava separately. The hepatic veins and the variants of their merging were more numerous than has been presented in anatomical textbooks. The variations in the numbers of hepatocaval confluences were characteristic especially of the right, but not of the left, divisional veins. In all the analysed corrosion casts, a substantial accessory middle hepatic vein from the right part of the right medial lobe was noted. Thus, only blood from the left part of the right medial and quadrate lobes was collected via the large-sized middle hepatic vein. The veins from the central division were relatively constant in their numbers, locations and drainage territories. In this regard, our findings provide a more comprehensi- ve understanding of the canine hepatic vein branching patterns, and they offer new anatomical perspectives for liver imaging diagnostic procedures and various hepatic surgical techniques.

\section{ACKNOWLEDGEMENTS}

The authors would like to thank American Journal Experts for proofreading the English usage in the manuscript. We acknowledge funding from the Slovenian Research Agency program P4-0053.

\section{REFERENCES}

1. Bismuth $H$ (1982) Surgical anatomy and anatomical surgery of the liver. World J Surg, 6: 3-9.

2. Carlisle C, Heath J (1995) Anatomy of the portal and hepatic veins of the dog: a basis for systematic evaluation of the liver by ultrasonography. Vet Radiol Ultrasound; 36: 227-333.

3. Covey JL, Degner DA, Jackson AH, Hofeling AD, Walshaw R (2009) Hilar liver resection in dogs. Vet Surg, 38: 104-111.

4. Dyce KM, Sack WO, Wensing CJG (2010) Textbook of veterinary anatomy. 4th $\mathrm{Ed}$. Saunders, London.

5. Evans HE, de Lahunta A (2013) Miller's anatomy of the dog4th Ed. Saunders, London.

6. Gadžijev EM, Ravnik D (1996) Atlas of applied internal liver anatomy. Springer-Werlag, Wien.

7. Hribernik M, de Cecchis L, Trotovšek B, Gadžijev EM, Ravnik D (2003) Anatomical variations of the right hepatic veins and their relevance to surgery. Hepatogastroenterology, 50: 656-660.

8. Kalt DJ, Stump JE (1993) Gross anatomy of the canine portal vein. Anat Histol Embryol, 22: 191-197.

9. Komtebedde J, Forsyth SF, Breznock EM, Koblik PD (1991) Intrahepatic portosystemic venous anomaly in the dog: perioperative management and complications. Vet Surg, 20: 37-42.

10. König HE, Liebich HG (2004) Veterinary anatomy of domestic mammals. Stuttgart, Parey Buchverlag.

11. Lamb CR (1991) Ultrasonography of the liver and biliary tract. Probl Vet Med, 3: 555-573.

12. Martin RA, Payne JT (1990) Angiographic results of intrahepatic portocaval shunt attenuation in three dogs. Sem Vet Med Surg, 5: 134-141.

13. Nickel R, Schummer A, Seiferle E (1996) Lerbuch der Anatomie der Haustiere: Band III. Parey Buchverlag, Berlin.

14. International Committee on Veterinary Gross Anatomical Nomenclature (2005) Nomina Anatomica Veterinaria. 5th Ed. Hannover, Columbia, Gent, Sapporo.

15. Schmidt S, Lohse CL, Suter PF (1980) Branching patterns of the hepatic-artery in the dog - arteriographic and anatomic study. Am J Vet Res, 41: 1090-1097.

16. Sleight DR, Thomford NR (1970) Gross anatomy of blood supply and biliary drainage of canine liver. Anat Rec, 166: 153-160.

17. Suter PF (1975) Portal vein anomalies in dog: their angiographic diagnosis. J Am Vet Radiol Soc, 16: 84-97.

18. Ursic M, Ravnik D, Hribernik M, Pečar J, Butinar J, Fazarinc G (2007) Gross anatomy of the portal vein and hepatic artery ramifications in dogs: corrosion cast study. Anat Histol Embryol, 36: 83-87.

19. Wittleben N (1989) Zur Topographie der Venen in der Leber des fetalen und neuegeborenen Hundes, Diss. Tierarzt. Fak. Ludwig-Maximillians-Universitat Munchen, Munchen. 